\title{
Automated Identification of Hard Exudates and Cotton Wool Spots using Biomedical image Processing
}

\author{
Sangramsing N. Kayte \\ Department of Computer \\ Science \& IT \\ Dr. Babasaheb Ambedkar \\ Marathwada University, \\ Aurangabad.
}

\author{
Raju S. Maher \\ Department of Computer \\ Science \& IT \\ Dr. Babasaheb Ambedkar \\ Marathwada University, \\ Aurangabad.
}

\author{
Charansing N. Kayte \\ Department of Computer \\ Science \& IT \\ Dr. Babasaheb Ambedkar \\ Marathwada University, \\ Aurangabad.
}

\begin{abstract}
The automatic identification of Image processing techniques for abnormalities in retinal images. Its very importance in diabetic retinopathy screening. Manual annotations of retinal images are rare and exclusive to obtain. The ophthalmoscope used direct analysis is a small and portable apparatus contained of a light source and a set of lenses view the retina. The existence of diabetic retinopathy detected can be examining the retina for its individual features. The first presence of diabetic retinopathy is the form of Microaneurysms. This research paper describes different works needed to the automatic identification of hard exudates and cotton wool spots in retinal images for diabetic retinopathy detection and support vector machine (SVM) for classifying images. This system is evaluated on a large dataset containing 129 retinal images. The proposed method Results show that exudates were detected from a database with $96.9 \%$ sensitivity, specificity $96.1 \%$ and $97.38 \%$ accuracy
\end{abstract}

\section{Keywords}

Diabetic retinopathy Retinal images, Biomedical image Processing, exudate, CAD.

\section{INTRODUCTION}

Diabetic Retinopathy (DR) is one of the most important causes of blindness and vision defects in developed countries [1]. Early detection is crucial for the prevention of visual loss and blindness in diabetes patients. In this disease, several visual retinal abnormalities appear in the retinal fundus, representing a visual indicator of changes in the vision [2]. The automatic detection of visual signs provides an actual way to obtain an early detection of diabetic retinopathy and to avoid future complications. In retinal images, early diabetic retinopathy lesions may be also classified into "red lesion" and "bright lesions", such as hard exudates and cotton wool spots. 3 The identification of these bright abnormalities acquiring manual remarks of these abnormalities in retinal images is a boring and time consuming task and its especially for large amounts of training data that are needed. The number of people afflicted with the disease continues to grow it. It occurs when the pancreas does not secrete enough insulin or the body is unable to process it properly. This results in an abnormal increase in the glucose level in the blood. Over time this high level of glucose causes damage to blood vessels. This damage affects both eyes and nervous system, as well as heart, kidneys and other organs [4].The diabetes have two types. Diabetic retinopathy is the most normal cause of new cases of blindness is an effect of diabetic retinopathy and its prevalence is set to continue rising. Estimated 50-65 new cases of blindness per 100,000 people happened every year
[3]. Microaneurysms and other areas of abnormal retinal blood vessels may leak fluid, causing the retina to bleed or swell. They are collapse of retinal blood vessels may result in fluid leaking into the center of the retina. Abnormal blood vessels that grow on the surface of the retina (neovascularization) which can bleed and scar.

Diabetic retinopathy is divided into some stages mild, moderate, severe and proliferative DR. A brief description of the different stages of DR.

a) Mild non-proliferative retinopathy: Microaneurysms, i.e., small swellings in the tiny blood vessels of the retina will be formed in this stage.

b) Moderate non-proliferative retinopathy: As the disease progresses, some blood vessels that nourish the retina are blocked.

c) Severe non-proliferative retinopathy: Many more blood vessels are blocked, depriving several areas of the retina of their blood supply. The affected areas of the retina begin to show sign of ischemia (lack of oxygen) such as blot hemorrhages, bleeding of the veins and intraretinal microvascular abnormalities.

d) Proliferative retinopathy: At this advanced stage, the nonproliferative factors produced by the retina begin to trigger the growth of new blood vessels. These new blood vessels are abnormal and fragile.

Progresses from mild non-proliferative abnormalities, characterized by increased vascular permeability, to moderate and severe non-proliferative diabetic retinopathy (NPDR), characterized by vascular closure, to proliferative diabetic retinopathy (PDR) [7]. An active learning framework has been used in different applications, such as detection of minelike objects in sonar imagery, labeling video data or contentbased information retrieval. In this paper, we propose a CAD system for the detection and differentiation of hard exudates, cotton wool spots in retinal images incorporating an active learner in the training process [8].

\section{METHOD}

In this work, 129 retinal photographs are used from DIARETDB0 a database including a selected of high-quality medical images which are representatives of the diabetic retinopathy and have been verified by experts for detection of moderate non-proliferative diabetic retinopathy (NPDR), severe NPDR, proliferative diabetic retinopathy and normal cases, have been studied. Feature extraction is the most important part of the proposed system. The inputs are used as extracted features to the classifiers. The first pre-processed 
images after a contrast enhancement process is carried out Feature extraction. Our pre-processing step primarily consists of image contrast improvement based on histogram equalization, morphological operators and followed by binarization [11]. The image resolution varied from $768 \times 576$ to $2048 \times 1536$ pixels while the field of view coverage varied 50 degree. From this data set, three images containing hard exudates, cotton wool spots the initial training set.

A human observer, performed manual annotations of hard exudates, cotton wool spots.10 unseen images were selected as the test set to evaluate the final algorithm performance. These images contained 98 hard exudates, 22 cotton wool spots. In the candidate extraction step, the objects that are possible bright lesions are extracted from the images using a earlier described technique. 3 The green channel of the RGB image is convolved with 14 digital filters based on Gaussian derivatives filter[6]. These filters are variation and translation of the image and were selected from a larger set of second order irreducible invariants using a feature selection algorithm [3]. This system is an iterative procedure where at each iteration the active learner is called to select an unlabeled sample from a pool of unlabeled data and an expert is asked for its label The next step is to classify each candidate as hard exudate, cotton wool spots incorporating an morphological techniques in the training process. The idea is to select efficiently a set of training samples from the unlabeled data in an active way to boost the performance of the classifier and reduce the number of samples that need to be labeled.

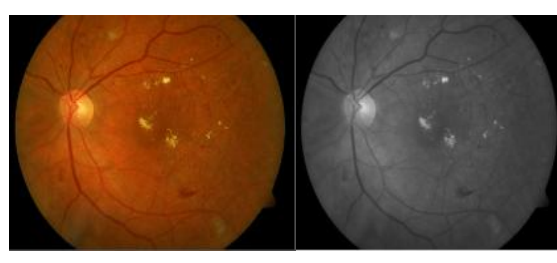

(a)

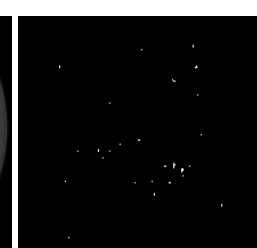

(c)
Figure 1(a) Original fundus image (b) Green Channel Image (c) lesson detection.

These features represent visual concepts that the ophthalmologists use to differentiate among the retinal lesions. The features provides information about the size, shape, color and contrast of the candidates, as well as background information such as the nearness to the vessels and the proximity to the closest red lesion. To examine these values, algorithms that vessel segmentation [12] and perform red lesion classification were previously applied to the retinal images [11]. No feature selection algorithms were applied in the process.

Table 1. Each object features extracted of candidate.

\begin{tabular}{|l|l|}
\hline $\begin{array}{l}\text { Features } \\
\text { of images }\end{array}$ & Description \\
\hline 1 & Area (size in pixel). \\
\hline 2 & Length of the perimeter of the candidate. \\
\hline 3 & Distance to the closest red lesion. \\
\hline
\end{tabular}

For the classifier c, we choose a four-class linear discriminant classifier. This classifier sets linear decision boundaries between the different classes in order to separate hard exudates, cotton wool spots, drusen and non-lesions. As the dimensionality of the problem is high, the selection of a linear classifier permits obtaining adequate classification performance as well as reducing time complexity.The final algorithm for the training process in the classification of candidates as hard exudates, cotton wool spots After applying the KNN algorithm, a classifier typically trained for the identification of(bright lesson) hard exudates, cotton wool spots is obtained.

\section{EXPERIMENTS AND RESULTS 3.1 Experiments}

The proposed detection methods are tested and evaluated on DIARETDB1 [12], a publicly available database of colored fundus images and corresponding ground truth images. Lesion based evaluation and image based evaluation are employed to measure the accuracy of the proposed detection method at the pixel level. Figure shows the results of microaneurysms and exudates detection for normal DR. In example the green component, of the RGB fundus image, was chosen to obtain the microaneurysms. Similar to the exudates detection algorithm, first the prominent structures within retina images, Such as blood vessel tree and optic disc are to be removed.

Table 2. Lesion based result.

\begin{tabular}{|c|c|}
\hline Classifier & KNN \\
\hline Total Images & 129 \\
\hline True positives & 87 \\
\hline False positives & 2 \\
\hline False negatives & 4 \\
\hline True negatives & 37 \\
\hline Sensitivity & $94 \%$ \\
\hline Specificity & $94.87 \%$ \\
\hline Accuracy & $95.38 \%$ \\
\hline
\end{tabular}

Several parameters such as True Positive (TP), True Negative (TN), False Positive (FP) and False Negative (FN) are calculated. These parameters are calculated by comparing the classifier outcome with the number of normal and abnormal images from the database. For an abnormal image, the result is true positive if the outcome of classification is abnormal and the result is False Negative (FN) if the classifier output is normal. For normal image, the result is True Negative (TN), if the classifier output is normal and False Positive (FP) if the classification outcome is abnormal. In a given image dataset, these parameters, TP,TN, FP, FN are used in the calculation of the accuracy, Sensitivity (SN) and specificity (SP)[2]. Performance of the classifier can be measured in terms of sensitivity, specificity and accuracy. The results were compared with the performance obtained using random sampling. This method randomly selects the next sample from the pool of unlabeled data. It relates to a passive learning model where the training set is a casual sampling of the data.

\subsection{Results}

The results for each performance of the sampling bright lesion detection method is good even for lesion based evaluation, as the proposed hybrid microaneurysm detection method resulted in a very high sensitivity with reasonable specificity, an 
ophthalmologist can take its assistance in detecting Microaneurysms, exudates and cotton wool spot in the mass screening of diabetic retinopathy. It achieves a sensitivity of $94 \%$ and a specificity of $94.87 \%$ and accuracy of $95.38 \%$, the performance of the microaneurysm detection method can be enhanced further by augmenting the amount of training data for the microaneurysm candidate object classification.

\section{DISCUSSION}

This paper presents an automated method to detect microaneurysm and exudates and cotton wool spots in digital fundus images. A new candidate microaneurysm detection scheme based on matched filtering and local relative entropy is proposed algorithm selected representative samples from database. The performance of this microaneurysm detection method is compared with mathematical morphology based microaneurysm detection method. A detection scheme that combines both detection methods is tested as well as detect. The results of the method on a per image basis show that the hybrid detection scheme achieved an accuracy of $95.38 \%$, sensitivity of $94 \%$ combined with $94.87 \%$ specificity. It indicates a large decrease in retinal images that have to be shown to an ophthalmologist.
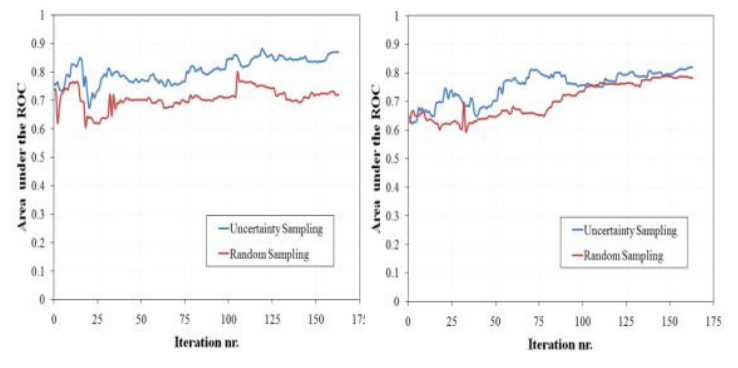

(a) (b)
Figure 2. ROC for the classification of (a) hard exudates;

\section{( b ) cotton wool spots.}

Fig. 2 shows that the active learning system outperformed random sampling in the identification of the three abnormalities because more informative samples were selected with the former algorithm that those that were picked randomly, the active learning selected more samples of the different classes, obtaining a balanced training set. Thus, this training approach provides a high benefit when the classification problem presents infrequent classes because more representative samples from these classes can be picked from the pool of unlabeled data. Therefore, active learning can also be seen as a form of data mining on the training database.

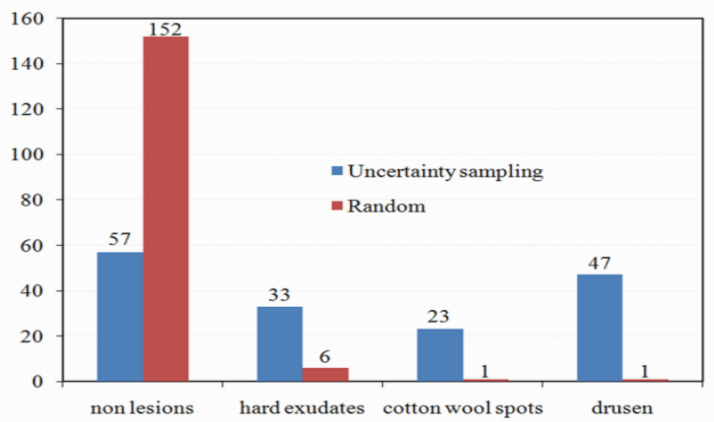

Figure 3. Compare sampling function and the random sampling function.
Uncertainty sampling was used as the query function to select the samples that need to be query next. This exploitation learning samples the region in the vicinity of the decision boundary, where the classifier is least confident. Figure 4 depicts some examples of the selected objects. These are hard cases to classify, which supports the notion that the active learner selects samples close to its decision boundary. There exists other query functions, called explorative learning, which sample from regions where no labeled samples are presented. 4 It could be interesting to apply an approach using alternatively exploitation and explorative learning to sample in different regions of the sample space.

\section{CONCLUSIONS}

This paper presents an automated method to detect microaneurysm and exudates in digital fundus images. A new candidate microaneurysm detection scheme based on matched filtering and local relative entropy is proposed. The performance of this microaneurysm detection method is compared with mathematical morphology based microaneurysm detection method this permits the design of a classifier with automatically selected useful samples. Results show that the proposed method increases the classification accuracy compared to random sampling. Local relative entropy the number of labeled examples required labeled databases that are now available in many application areas. An ophthalmologist can take its help in detecting microaneurysms, exudates and cotton wool spot in the mass screening of diabetic retinopathy.

\section{REFERENCES}

[1] Kinyoun, J., Barton, F., Fisher, M., Hubbard, L., Aiello, L., and Ferris, F., "Detection of diabetic macular edema. Ophthalmoscopy versus photography-Early Treatment Diabetic Retinopathy Study Report Number 5. The ETDRS Research Group." Ophthalmology 96, 746-750 (1989).

[2] Early Treatment Diabetic Retinopathy Study Research Group, "Early Photocoagulation for Diabetic Retinopathy: ETDRS report 9," Ophthalmology 98, 766785 (1991).

[3] Raju Sahebrao Maher, Sangramsing N. Kayte, Sandip T. Meldhe, Mukta Dhopeshwarkar, "Automated Diagnosis Non-proliferative Diabetic Retinopathy in Fundus Images using Support Vector Machine" International Journal of Computer Applications (0975 - 8887)Volume 125 - No.15, September 2015.

[4] Dura, E., Zhang, Y., Liao, X., Dobeck, G. J., and Carin, L., "Active learning for detection of mine-like objects in side-scan sonar imagery," IEEE Journal of Oceanic Engineering 30(2), 360-371 (2005).

[5] Yan, R., Yang, J., and Hauptmann, A., “Automatically labeling video data using multi-class active learning," in [Proc. Ninth IEEE International Conference on Computer Vision], 516-523 (2003).

[6] Zhang, C. and Chen, T., "An active learning framework for content-based information retrieval," IEEE Transactions on Multimedia 4(2), 260-268 (2002).

[7] Raju Sahebrao Maher, Dnyaneshwar S. Panchal, Sangramsing Kayte, Dr. Mukta Dhopeshwarkar" Automatic Identification of Various Stages of Diabetic Retinopathy Using Retinal Fundus Images" International Journal of Advanced Research in Computer Science and 
Software Engineering, Volume 5, Issue 9, September 2015.

[8] Abr'amoff, M. D. and Niemeijer, M., "The automatic detection of the optic disc location in retinal images using optic disc location regression," in [Engineering in Medicine and Biology Society, 2006. EMBS '06.] , 4432-4435 (2006).

[9] Dima, C. and Hebert, M., "Active learning for outdoor obstacle detection," in [Proceedings of Robotics: Science and Systems], (2005).

[10] Niemeijer, M., van Ginneken, B., Staal, J., SuttorpSchulten, M., and Abr'amoff, M. D., "Automatic detection of red lesions in digital color fundus photographs," IEEE Transactions on Medical Imaging 24(5), 584-592 (2005).

[11] Raju Maher, Sangramsing Kayte, Dnyaneshwar Panchal, Pankaj Sathe, Sandip Meldhe, "A Decision Support System for Automatic Screening of Non-proliferative Diabetic Retinopathy" International Journal of Emerging Research in Management and Technology, Volume4,Issue-10, October-2015

[12] Raju Maher, Dr.Mukta Dhopeshwarkar "Automated Detection of Non-proliferative Diabetes Retinopathy Using Fundus Images" International Journal of Advanced Research in Computer Science and Software Engineering Volume 5, Issue 3, March 2015. 